\title{
Revisão sistemática sobre fatores associados à relação entre habilidades sociais e suporte social
}

\author{
Vanessa Santiago Ximenes ${ }^{1}$ \\ Francine Náthalie Ferraresi Rodrigues Queluz ${ }^{2}$ \\ Elizabeth Joan Barham ${ }^{3}$ \\ ${ }^{1}$ Universidade Federal de São Carlos, SP, Brasil \\ https://orcid.org/0000-0003-4079-5525 \\ ${ }^{2}$ Universidade São Francisco, SP, Brasil \\ https://orcid.org/0000-0002-8869-6879 \\ ${ }^{3}$ Universidade Federal de São Carlos, SP, Brasil \\ https://orcid.org/0000-0002-7270-4918
}

\begin{abstract}
Resumo
Um repertório bem desenvolvido de habilidades sociais permite interações sociais de melhor qualidade. Assim, habilidades sociais afetam o acesso a suporte social, mas pouco se sabe sobre fatores que aumentam ou reduzem a eficácia das habilidades sociais na obtenção desse tipo de suporte. O objetivo deste estudo foi identificar fatores associados à magnitude da relação entre habilidades sociais e suporte social. Foram consultadas as bases de dados Scielo, Lilacs, Medline, PsycNET e Web of Science, recuperando artigos publicados em Espanhol, Inglês ou Português entre 1997 e 2017. Quinze artigos foram selecionados. A idade, o gênero, e a fonte de apoio foram alguns dos fatores que influenciaram a relação entre habilidades sociais e suporte social. Em pesquisas futuras, será importante testar um modelo da relação entre habilidades sociais e suporte social, incluindo estas variáveis intervenientes.
\end{abstract}

Palavras-chave: competência social, suporte social; qualidade de vida, assertividade.

\section{Revisión sistemática sobre factores asociados a la relación entre habilidades sociales y apoyo social}

\section{Resumen}

Un repertorio bien desarrollado de habilidades sociales permite interacciones sociales de mejor calidad. Así, habilidades sociales afectan el acceso al apoyo social, pero poco se sabe sobre factores que aumentan o reducen la eficacia de las habilidades sociales para obtener este tipo de soporte. El objetivo de este estudio fue identificar factores asociados a magnitud de la relación entre habilidades sociales y soporte social. Se han consultado las bases de datos Scielo, Lilacs, Medline, Web of Science y PsycNET para seleccionar artículos publicados en Español, Inglés o Portugués entre 1997 y 2017. Quince artículos han sido seleccionados. La edad, el género, y la fuente de apoyo fueron algunos de los factores que influenciaron la relación entre habilidades sociales y soporte social. En investigaciones futuras, será importante probar un modelo de la relación entre habilidades sociales y soporte social, incluidas las variables intervinientes.

Palabras clave: habilidades sociales, apoyo social, calidad de vida, asertividad. 


\title{
Systematic review of factors that influence the relationship between social skills and social support
}

\begin{abstract}
A well-developed repertoire of social skills permits higher quality social interactions. As such, social skills affect access to social support, but little is known about factors that increase or diminish the effectiveness of social skills in gaining this support. The objective of this study was to identify factors that influence the strength of the relationship between social skills and social support. The Scielo, Lilacs, Medline, PsycNET, and Web of Science databases were consulted, recovering articles published in Spanish, English or Portuguese, between 1997 and 2017. Fifteen articles were selected. Age, gender, and source of support were some of the factors that influenced the relationship between social skills and social support. In future studies, it will be important to test a model of the relationship between social skills and social support, that includes these intervening variables.
\end{abstract}

Keywords: social skills, social support, quality of life, assertiveness.

Em inúmeras circunstâncias, como quando o indivíduo está doente ou quando apresenta um número elevado de atividades diárias, é necessário ter o apoio de outras pessoas para gerenciar as demandas do dia a dia. A manutenção de relações interpessoais de qualidade favorece o processo de inserção e atuação em sociedade, não somente pela necessidade de convivência entre as pessoas, mas também devido à satisfação com a vida que tais vínculos podem gerar (Cardoso \& Baptista, 2015). Diversos pesquisadores demonstraram que um repertório bem desenvolvido de habilidades sociais (HS) repercute em benefícios às interações sociais, tornando-as de melhor qualidade e mais proveitosas (Carneiro, 2014; Del Prette \& Del Prette, 2017; Pinto, Barham, \& Del Prette, 2016; Queluz, Barham, Del Prette, \& Santos, 2018). O mesmo parece acontecer em relação ao suporte social (Cardoso \& Baptista, 2015). Estudiosos pontuam que vínculos sociais, quando coesos e consistentes, podem proporcionar melhor qualidade de vida e percepção de maior suporte social, elevando a autoestima e a satisfação com a vida entre aqueles que trocam experiências e sentimentos a partir da vinculação com redes de apoio (Hajek et al., 2016; Rodrigues \& Madeira, 2009). Considerando pesquisas recentes na área, realizadas em diferentes contextos, será possível analisar a magnitude da relação entre as HS e o suporte social, buscando identificar fatores que podem influenciar a eficácia da emissão de HS para obter ajuda, o que justifica a realização da presente revisão de literatura.

\section{Habilidades Sociais}

Segundo Del Prette e Del Prette (2017), as HS dizem respeito a classes de comportamentos sociais que são importantes para lidar de modo socialmente competente com as interações interpessoais. Além das HS, as interações sociais bem-sucedidas dependem da competência social. Enquanto as HS envolvem um conjunto de comportamentos sociais que são emitidos durante interações com outras pessoas, a competência social diz respeito à funcionalidade do desempenho social do indivíduo, pois envolve os efeitos desse desempenho nas relações interpessoais a partir de um determinado contexto (Del Prette \& Del Prette, 2017). Outra característica relevante das HS é que elas são aprendidas, fato que possibilita, por meio do treinamento em HS, o ensino e o refinamento de comportamentos indispensáveis a uma interação social de qualidade em um determinado ambiente social (Braz, Cômodo, Del Prette, Del Prette, \& Fontaine, 2013; Del Prette \& Del Prette, 2017). Assim, segundo Del Prette e Del Prette (2017), indivíduos que apresentam um repertório bem desenvolvido em diferentes classes de HS, como assertividade, comunicação, empatia, civilidade e expressão de sentimentos positivos, e têm um desempenho socialmente competente tendem a emitir comportamentos que constroem e mantêm interações sociais bem-sucedidas, considerando as próprias demandas e as das pessoas com quem se relacionam.

A relação entre pais e filhos pode ser palco de importantes interações sociais que, a depender de sua adequabi- 
lidade, podem ser alavancas para o desenvolvimento das HS em crianças. Práticas parentais adequadas, com regras consistentes e equilíbrio entre restrição (respeitar o outro) e permissividade (seguir interesses pessoais), oportunizam um ambiente propício para a aprendizagem de HS (Murta, Del Prette, \& Del Prette, 2013). Além disso, uma consequência positiva de um repertório bem desenvolvido em HS, mencionada em estudos, foi que mães tendem a manter relações de maior intimidade com os filhos ao conversarem, expressarem sentimentos e estabelecerem limites, contribuindo para o aprimoramento de comportamentos sociais de seus filhos (Braz et al., 2013; Cômodo, Del Prette, \& Del Prette, 2017). Por outro lado, um relacionamento familiar de baixa qualidade, envolvendo punição corporal (Martins et al., 2014) e comunicação deficitária, pode comprometer a construção do repertório de HS em crianças, gerando consequências negativas, evidenciadas principalmente nas interações da criança com seus pares (Stasiak, Weber, \& Tucunduva, 2014).

Conforme afirmaram Hajek et al. (2016), as relações interpessoais de qualidade podem ser fatores de proteção e promoção de saúde. No estudo de Malinauskas et al. (2014), com 400 adolescentes lituanos, as HS e a satisfação com a vida estiveram positivamente relacionadas, destacando o efeito protetor das HS. Resultados convergentes aos de Malinauskas et al. (2014) foram encontrados em pesquisas que têm como enfoque as HS voltadas para a população idosa (Braz et al., 2013; Carneiro \& Falcone, 2016; Carneiro, 2014), sendo que um repertório refinado de HS por parte de idosos apresentou associação positiva com qualidade de relações intergeracionais e relação negativa com depressão. Em contrapartida, déficits em HS e relacionamentos interpessoais conflituosos são considerados fatores de risco para vulnerabilidade emocional, ansiedade e sobrecarga emocional (Bolsoni-Silva \& Loureiro, 2014; Giron \& Souza, 2015; Pinto, 2016; Queluz et al., 2018).

Logo, integrando os dados de pesquisas, é possível afirmar que as HS podem fortalecer as relações interpessoais ao "munir" os indivíduos com compor- tamentos socialmente adequados para se relacionarem entre si. Desse modo, as HS podem ser adquiridas e refinadas ao longo da vida, de modo a promover percepções de melhor qualidade de vida e satisfação com os relacionamentos sociais, podendo contribuir para a formação das redes de suporte (Del Prette \& Del Prette, 2017; Pinto \& Oliveira, 2015; Pinto, 2016).

\section{Suporte social}

O conceito de suporte social pode ser analisado a partir de diferentes contextos de interações humanas, tais como a família, o trabalho, a religião, o ensino, entre outros (Cardoso \& Baptista, 2015). Neri (2014) definiu redes de apoio ou suporte como conjuntos hierárquicos de indivíduos que mantêm entre si relações de reciprocidade, sendo que sua estrutura, funções e desejabilidade se modificam a depender da idade, das necessidades e do grau de dependência dos membros da rede de apoio. Já o suporte social ou apoio social é um produto da atuação das relações sociais e da percepção do indivíduo sobre a qualidade, a frequência e a adequação das ajudas que lhe são oferecidas, a partir das suas necessidades (Neri, 2014). No presente estudo o termo suporte social será utilizado também para se referir a apoio social, rede de apoio e rede de suporte, pois mesmo não sendo sinônimos, descrevem relações de ajuda ao outro.

Neri (2014) pontuou, ainda, que o suporte social proporciona benefícios importantes, como: diminuição nos riscos de desenvolvimento de enfermidades, mais chances de recuperação frente a doenças, percepção de melhor bem-estar e adaptação diante de situações que geram estresse. Em consonância com essas afirmações, uma série de pesquisadores reportou relações positivas entre o suporte social e indicadores de bem-estar (Hill, 2016; Mackin, Perlman, Davila, Kotov, \& Klein, 2017; Santos, 2016). Hill (2016) afirmou que o suporte social emocional foi preditivo de melhor qualidade de vida e menor índice de depressão. Em Mackin et al. (2017), os autores descobriram que o suporte familiar foi um dos fatores de proteção contra os efeitos deletérios do 
estresse em 550 adolescentes. Santos (2016) pontuou que os indicadores que mais favoreceram a satisfação com a vida para as mulheres foram o apoio familiar e de pares. Já para o sexo masculino, o suporte social de pares contribuiu para a melhor satisfação com a vida (Santos, 2016). Em pesquisas com idosos, os resultados corroboraram àqueles encontrados com participantes adolescentes e adultos. Idosos com bom nível de suporte social se perceberam com maior satisfação com a vida, menor estresse e melhor qualidade de vida (Carneiro, 2014; Hajek et al., 2016).

Dessa forma, dada a centralidade das HS e do suporte social para a saúde e a qualidade das interações sociais e considerando: (a) a importância das HS enquanto prováveis mediadoras da aquisição e manutenção das redes de apoio e (b) a necessidade de se averiguar fatores que podem influenciar a magnitude da relação entre essas variáveis, este estudo teve como objetivo investigar pesquisas nacionais e internacionais nas quais foi avaliada a relação entre as HS e o suporte social, além de verificar a magnitude desssas relações e fatores associados com a força dessas relações. Justifica-se a elaboração do presente estudo a partir da carência de integração e de sistematização de dados que relacionem as HS e o suporte social, assim como da verificação e da comparação da magnitude de suas correlações. A hipótese norteadora desse estudo foi de uma correlação positiva entre as HS e o suporte social, com correlações de magnitude fraca a moderada, uma vez que, embora ambos construtos tenham uma contribuição positiva para o bem-estar geral de diferentes populações (Del Prette \& Del Prette, 2017; Hajek et al., 2016; Mackin et al., 2017), espera-se que outros fatores modifiquem a força desta relação.

\section{Método}

O levantamento bibliográfico foi operacionalizado mediante a procura eletrônica de artigos nas bases de dados Scielo, Lilacs, Medline, PsycNET e Web of Science, entre os anos de 1997 e dezembro de 2017. Os seguintes descritores foram utilizados: habilidade(s) social(is), competência social e assertividade. Eles foram cruzados com apoio social, suporte social, rede de suporte social e suporte familiar, nos idiomas português, inglês e espanhol. Habilidades sociais, competência social e assertividade não são conceitos considerados sinônimos, porém, são termos usados em estudos para descrever habilidades relacionadas a comportamentos sociais e, por isso, foram agrupados e utilizados enquanto descritores. $\mathrm{O}$ mesmo pode ser entendido em relação a apoio social, suporte social, rede de suporte social e suporte familiar, uma vez que não são sinônimos, mas são termos usados por pesquisadores para se referirem a contextos no quais ajuda é recebida de outras pessoas. Os artigos foram selecionados a partir dos seguintes critérios de inclusão: (a) idioma de publicação: estudos publicados em pPortuguês, inglês e espanhol; (b) ano de publicação: trabalhos publicados entre 1997 e dezembro de 2017, somando, portanto, um intervalo de 20 anos; (c) modalidade de produção científica: foram incluídos artigos originais de relatos de pesquisas empíricas de caráter quantitativo, transversal e de delineamento correlacional em periódicos avaliados por pares; e (d) descritores: foram selecionados artigos que apresentaram os descritores desta revisão no título ou no resumo.

Os seguintes critérios de exclusão foram adotados: (a) estudos nos quais a correlação entre as HS e o suporte social não foi reportada e; (b) estudos realizados em contextos que envolviam relações assimétricas (por exemplo, funcionários de empresas ou professores escolares), pois nesses ambientes havia o pagamento de pessoas para oferecer apoio aos demais. Após a seleção inicial dos artigos, e considerando os critérios de inclusão e exclusão, dois juízes independentes avaliaram os resumos para selecionar o escopo do presente estudo, discutindo diferenças de opinião até chegarem a um consenso (Anfara, Brown, \& Magione, 1992). Adiante, foram recuperados os textos completos dos estudos selecionados, formalizando o material de análise desta revisão sistemática da literatura. 
No total, foram lidos 725 resumos de estudos científicos, sendo 369 artigos na Scielo, 113 na Lilacs, 88 na Medline, 122 na PsycNET e 33 na Web of Science. Em um primeiro momento, foram excluídos artigos nos quais não foi reportada a correlação entre as HS e o suporte social (494 estudos), outros que tiveram como foco interações em um contexto formal (106 estudos no contexto escolar e 8 estudos no contexto organizacional), artigos em idiomas diferentes daqueles previamente estabelecidos (55 estudos), artigos que não disponibilizaram o acesso integral ao texto (26 estudos) via Capes Periódicos, e pesquisas duplicadas, ou seja, que apareceram em mais de uma base de dados (21 estudos). Desta forma, foram selecionados 15 artigos que atenderam a todos os critérios de inclusão e exclusão. Na Figura 1, apresenta-se um diagrama do processo de busca e seleção dos artigos da presente revisão.

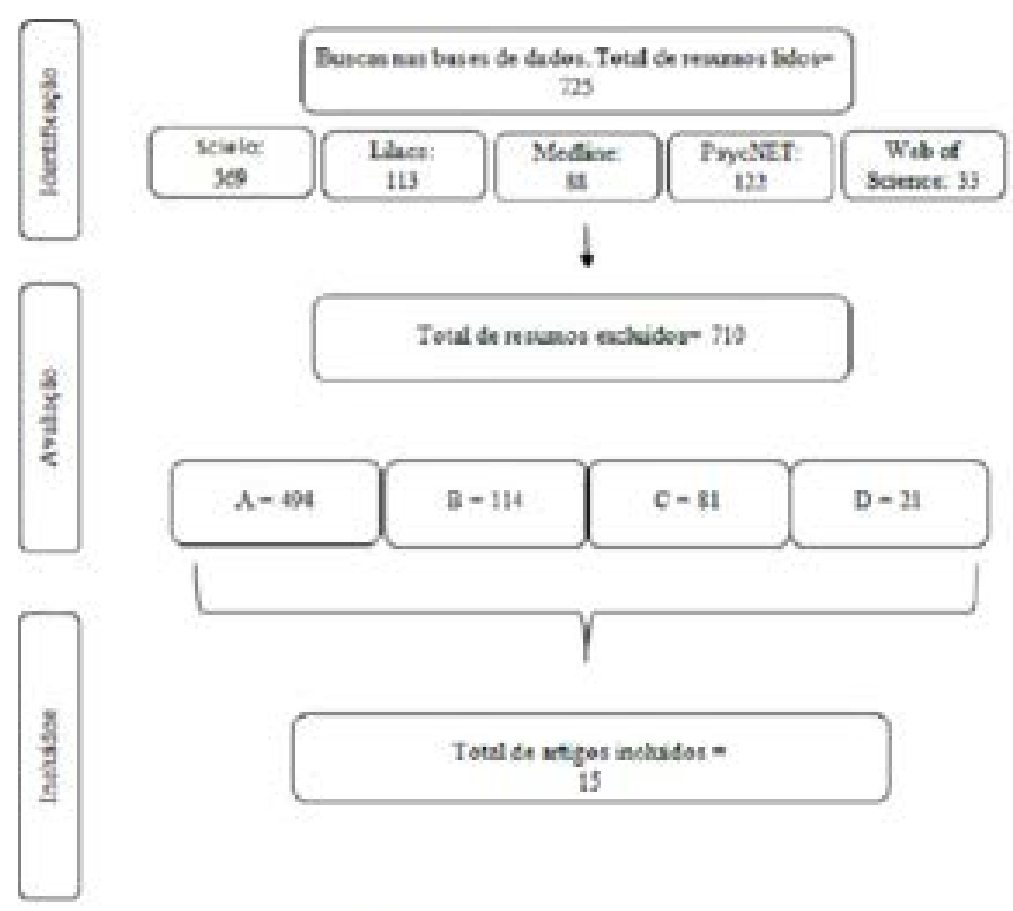

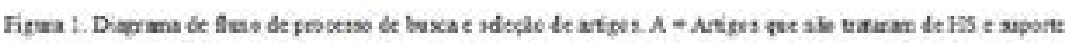

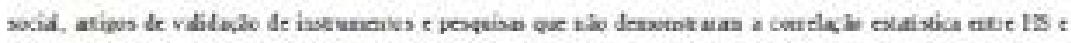

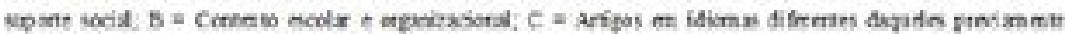

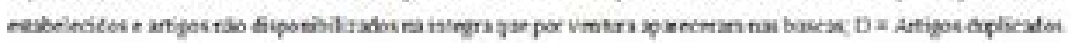


Com o objetivo de organizar o material encontrado, foram criadas três dimensões de análise, sendo elas: (a) descrição sumária da amostra; (b) variáveis investigadas ; e (c) principais relações entre as variáveis investigadas. Tais dimensões permitiram a sistematização dos dados e facilitaram a compreensão das informações.

\section{Resultados e discussão}

Houve prevalência de artigos conduzidos nos Estados Unidos (cinco estudos). Pesquisas realizadas no Brasil, no Canadá, na Turquia, na Suécia, na Suíça, no Japão, na Alemanha, na Noruega, na Espanha e na Argentina completaram a presente revisão com um estudo de cada país. Observa-se que a associação entre as HS e o suporte social vem sendo pesquisada em distintas nacionalidades, demonstrando que pesquisadores de vários países identificaram a necessidade de investigar variáveis que facilitam a obtenção de suporte social.

Quanto à composição das amostras, a maioria dos estudos foram realizados com adultos (sete estudos) (Dierk et al., 2006; García-Bóveda et al., 2007; Kukulu et al., 2006; McIntosh et al., 2016; Müller et al., 2015; Riggio \& Kwong, 2009; Schoenrock et al., 1999), seguida por quatro estudos com participantes adolescentes de 11 a 18 anos (Bédard et al., 2014; Eskin, 2003; Nilsen Karevold, Røysamb, Gustavson, \& Mathiesen, 2013; Segrin, McNelis, \& Swiatkowski, 2016), duas pesquisas com crianças (Takahashi, Okada, Hoshino, \& Anme, 2015; Vázquez \& Lemos, 2013) e outras duas com a população idosa (Carneiro et al., 2007; Segal, 2005). O maior número de estudos com adultos pode refletir mais autonomia e independência de indivíduos nessa fase da vida, diferentemente de crianças, que tendem a depender de outras pessoas para, por exemplo, serem autorizados a participar de pesquisas, se deslocarem até o local de coleta de dados, entre outros.

Com base no fato de que a relação entre as HS e o suporte social vem sendo investigada em populações com distintas faixas etárias, torna-se possível atentar para a existência de particularidades próprias de cada etapa de vida na emissão de comportamentos socialmente competentes e na obtenção do tipo de suporte necessário para enfrentar conflitos e resolver problemas cotidianos. Por exemplo, uma pessoa idosa pode precisar de habilidades sociais para fazer amizades novas, após perder familiares e amigos de sua faixa etária. Um adulto mais novo pode usar a mesma habilidade para se estabelecer em um emprego ou para lidar com um papel social novo na sua vida pessoal. Esse achado está de acordo com a afirmação de Del Prette e Del Prette (2017) de que as HS são situacionais e em cada período da vida serão necessárias HS específicas para promover interações sociais de qualidade.

Para além da relação entre HS e suporte social, alguns pesquisadores dos estudos desta revisão investigaram a associação desses construtos e outras variáveis apresentadas nos artigos revisados, como depressão e qualidade de vida. Na Tabela 1, são apresentadas as variáveis dependentes e os estudos em que foram incluídas.

\section{Tabela 1}

Variáveis investigadas

\begin{tabular}{|c|c|}
\hline Variáveis & Estudos \\
\hline Depressão & $\begin{array}{l}\text { Bédard et al., 2014; Carneiro et al., 2007; } \\
\text { McIntosh et al., 2016; Müller et al., 2015; } \\
\text { Nilsen et al.,2013; Segal, 2005; Segrin et al., } \\
2016\end{array}$ \\
\hline Evitação social & Bédard et al., 2014 \\
\hline Estresse & McIntosh et al., 2016; Segrin et al., 2016 \\
\hline Ansiedade & Bédard et al., 2014; McIntosh et al., 2016 \\
\hline Solidão & Riggio e Kwong, 2009; Segrin et al., 2016 \\
\hline Paranoia & Riggio e Kwong, 2009 \\
\hline Bem-estar & Dierk et al., 2006 \\
\hline Qualidade de vida & Carneiro et al., 2007; Müller et al., 2015 \\
\hline Automonitoramento & Schoenrock et al., 1999 \\
\hline
\end{tabular}


Em nove estudos, os autores relataram que as HS e o suporte social estavam positivamente associados com indicadores de bem-estar e negativamente relacionados à depressão, ansiedade, evitação social, estresse, solidão e paranoia, corroborando os achados de pesquisadores que estudaram esses construtos individualmente (BolsoniSilva \& Loureiro, 2014; Braz et al., 2013; Carneiro \& Falcone, 2016; Giron \& Souza, 2015; Hill, 2016; Mackin, et al., 2017; Malinauskas et al., 2014; Pinto, 2016; Queluz et al., 2018; Santos, 2016). Tal resultado enfatiza a importância das HS e do suporte social enquanto variáveis que influenciam uma melhor qualidade de vida. Isto é, um repertório desenvolvido de HS eleva a probabilidade de o indivíduo emitir comportamentos desejados durante uma interação social e, assim, construir e manter interações bem-sucedidas e fundamentadas em um desempenho socialmente competente (Del Prette \& Del Prette, 2017; Pinto et al., 2016). A partir desses vínculos de qualidade, a pessoa pode angariar apoio emocional, instrumental e informacional, condição que aumenta a probabilidade de que ela seja capaz de solucionar ou amenizar impasses que geram estresse e perceba mais positivamente a sua qualidade de vida (Hajek et al., 2016; Pinto et al., 2016).

Concomitantemente, o suporte social pode, também, ser revertido em interações que ajudem o indivíduo a se sentir menos sozinho e mais socialmente competente, gerando sentimentos de autoestima e autoconfiança, que também contribuem para maior qualidade de satisfação com a vida (Carneiro, 2014; Pinto et al., 2016). Assim, deve-se estudar o efeito das HS e do suporte social em distintas variáveis a fim de entender a conexão entre essas medidas e contribuir para a promoção estratégias de intervenção - embasadas nessas variáveis - que proporcionem maior qualidade de vida às pessoas a partir de interações sociais reforçadoras, que foi um dos focos do presente estudo.

$\mathrm{Na}$ Tabela 2, são demonstradas as magnitudes das correlações encontradas. Salienta-se que os estudos de Segrin et al. (2016) e McIntosh et al. (2016) não constam na Tabela 2, por não apresentarem valores de correlação bivariada e sim de beta $(\beta)$, por envolverem análises de regressão, as quais permitem avaliar a contribuição das HS, em conjunto com outros fatores, para explicar a obtenção de suporte social. Tais estudos foram mantidos na presente revisão por também demonstrarem relações estatísticas significativas entre as variáveis em estudo (HS e suporte social). Em Segrin et al. (2016), os autores encontraram um valor de $\beta=0,59(p<0,001) \mathrm{e}$ em McIntosh et al. (2016), $\beta=0,51(p<0,001)$, no qual as HS atuaram como variável independente e preditora de maior percepção de suporte social.

\section{Tabela 2}

Magnitude das correlaç̃̃es reportadas

\begin{tabular}{|c|c|c|c|}
\hline Autores & Amostra & Variáveis & Correlação (r) \\
\hline Schoenrock, et al. (1999) & Adolescentes & Competência Social x Suporte Familiar & $\begin{array}{c}0,38(\mathrm{~m})^{* *} \\
0,24(\mathrm{f})^{* *}\end{array}$ \\
\hline \multirow{4}{*}{ Eskin (2003) } & & Assertividade x Suporte de Pares (Suecos) & $\begin{array}{l}0,51(\mathrm{~m}) * * * \\
0,38(\mathrm{f}) * * *\end{array}$ \\
\hline & Adolescentes & Assertividade x Suporte de Pares (Turcos) & $\begin{array}{l}0,33(\mathrm{~m}) * * * \\
0,36(\mathrm{f}) * * *\end{array}$ \\
\hline & & Assertividade x Suporte Familiar (Suecos) & $\begin{array}{c}0,24(\mathrm{~m})^{* * *} \\
0,16(\mathrm{f})^{*}\end{array}$ \\
\hline & & Assertividade x Suporte Familiar (Turcos) & $\begin{array}{l}0,16(\mathrm{~m})^{*} \\
0,09(\mathrm{f})^{* *}\end{array}$ \\
\hline
\end{tabular}




\begin{tabular}{|c|c|c|c|}
\hline Segal (2005) & $\begin{array}{l}\text { Idosos } \\
\text { institucionalizados }\end{array}$ & Assertividade x Suporte Social & 0,03 \\
\hline Kukulu, et al. (2006) & $\begin{array}{l}\text { Universitários (em } \\
\text { enfermagem) }\end{array}$ & Assertividade x Suporte Familiar & $0,23^{* *}$ \\
\hline \multirow{4}{*}{ Dierk, et al. (2006) } & & Medo de críticas x Suporte Social & $-0,54^{*}$ \\
\hline & Adultos obesos & Medo de contato social x Suporte Social & $-0,56^{*}$ \\
\hline & & Tomar decisões x Suporte Social & $0,44^{*}$ \\
\hline & & Incapacidade de negar pedidos x Suporte Social & $-0,35^{*}$ \\
\hline García-Bóveda, et al. (2007) & $\begin{array}{l}\text { Adultos com } \\
\text { esquizofrenia }\end{array}$ & HS x Tamanho da Rede de Suporte Social & $0,29^{*}$ \\
\hline Carneiro, et al. (2007) & Idosos & HS x Suporte Social & $0,26^{*}$ \\
\hline Riggio \& Kwong (2009) & Jovens adultos & HS x Tamanho da Rede de Suporte Social & $0,23^{*}$ \\
\hline Vázquez \& Lemos (2013) & Crianças com câncer & HS x Suporte Social & $0,53^{*}$ \\
\hline Nilsen, et al. (2013) & Adolescentes & HS x Suporte Familiar & $\begin{array}{c}0,31(\mathrm{~m})^{*} \\
0,25(\mathrm{f})^{*}\end{array}$ \\
\hline \multirow{2}{*}{ Bédard, et al. (2014) } & Adolescentes & Competência Social x Suporte de Pares & $\begin{array}{c}0,57(\mathrm{~m})^{*} \\
0,48(\mathrm{f})^{*}\end{array}$ \\
\hline & & Competência Social x Suporte Familiar & $\begin{array}{c}0,27(\mathrm{~m})^{*} \\
0,03(\mathrm{f})^{*}\end{array}$ \\
\hline \multirow{4}{*}{ Takahashi, et al. (2015) } & & HS (Assertividade) x Suporte Parental (crianças com 2 anos) & 0,04 \\
\hline & & HS (Assertividade) x Suporte Parental (criança com 3 anos) & 0,05 \\
\hline & Crianças & HS (Assertividade) x Suporte Parental (criança com 4 anos) & $0,10^{*}$ \\
\hline & & HS (Assertividade) x Suporte Parental (criança com 5 anos) & $0,17^{*}$ \\
\hline Müller, et al. (2015) & $\begin{array}{l}\text { Adultos com lesão } \\
\text { medular }\end{array}$ & HS x Suporte Social & $0,27^{*}$ \\
\hline
\end{tabular}

Notas. $* p<0,05 ; * * p<0,01 ; * * * p<0,001 . \mathrm{m}=$ participantes do sexo masculino. $\mathrm{f}=$ participantes do sexo feminino.

De acordo com as informações apresentadas na Tabela 2, nota-se que, para avaliar comportamentos sociais (adequados) nas interações sociais, os pesquisadores utilizaram medidas de competência social, HS, assertividade e classes específicas de HS (como receber críticas, manter contato social, negar pedidos e tomar decisões). Quanto ao suporte social, pode-se observar que ele foi avaliado a partir do suporte familiar, o suporte de pares e com base no tamanho da rede de suporte social. Assim, ora existiu uma distinção apenas na fonte do fenômeno estudado (como é o caso de suporte social familiar e suporte social de pares) e ora existiu diferença na variável investigada (como ocorre com a competência social e as HS, que não são entendidas enquanto sinônimos). Portanto, as informações da Tabela 2 permitem somente uma comparação geral entre os resultados dos diferentes estudos. 
Pode-se afirmar que a maior parte das correlações foi de magnitude variando entre fraca $(0,20-0,39) \mathrm{e}$ moderada (entre 0,40 e 0,59), segundo a definição de Levin e Fox (2004), demonstrando que um repertório comportamental desenvolvido de HS está relacionado com a percepção de suporte social. Nota-se que Dierk et al. (2006) observaram algumas relações negativas, um vez que avaliaram déficits de habilidades sociais. Portanto, esses resultados estão de acordo com a hipótese inicial, uma vez que ao relacionar os dois construtos (HS e suporte social), esperava-se que as correlações fossem de magnitude fraca a moderada por se tratarem de construtos psicológicos, nos quais há outras variáveis envolvidas que podem afetar a variância comum entre os dois construtos (Pasquali, 2015). As correlações positivas mais altas foram encontradas entre: competência social e suporte de pares, em adolescentes $(r=0,57 ; p<0,01)$ (Bédard, et al., 2014), e as HS e o suporte social das crianças participantes do estudo de Vásquez e Lemos (2013) $(r=0,53 ; p<$ $0,01)$. Percebe-se, então, que as correlações mais fortes foram encontradas em um grupo etário mais novo, indicando que diferenças nas habilidades de usar as HS em crianças e jovens podem exercer uma influência significativa na sua capacidade de obter suporte social. Ainda de acordo com estudos desta revisão, a relação entre as HS e o suporte social de pares demonstrou ser mais expressiva quando comparada ao suporte familiar (Bédard et al., 2014; Eskin, 2003).

Considerando que crianças e adolescentes estão em desenvolvimento (motor, cognitivo, emocional e social) (Del Prette \& Del Prette, 2017), levanta-se a hipótese de que, no geral, familiares assumem a responsabilidade de suprir as necessidades práticas e financeiras desses, com pouco efeito das HS da criança sobre a obtenção desse apoio. Portanto, nas suas interações com pares, é provável que jovens procurem, principalmente, apoio socioemocional. Em contraste, os adultos estudados viviam em contextos de vulnerabilidade e dependência, de forma que é provável que possuíam necessidades significativas de apoio socioemocional, prático e financeiro, resultando em maior probabilidade de pessoas na sua rede de suporte não terem condições de suprir as suas necessidades. Assim, diferenças nos tipos e na disponibilidade do apoio que as crianças e adultos buscavam podem ter contribuído para uma correlação de maior magnitude entre as HS e medidas de suporte social entre os jovens do que entre os adultos (Giron \& Souza, 2015). Ademais, em interações com pares, as pessoas tendem a ser mais sensíveis à emissão de comportamentos socialmente competentes do que em interações com membros da própria família, tendo em vista que é necessário que os envolvidos construam voluntariamente relações extrafamiliares. Portanto, na interação com pares, é crucial a emissão de habilidades sociais, como iniciar e manter conversação, expor ideias e expressar sentimentos, para criar tal vínculo social (Pinquart \& Sörensen, 2000), enquanto nas famílias, o apoio tende a ser oferecido em função de necessidades e não das habilidades dos envolvidos (Cômodo et al., 2017).

Apesar de existir uma relação mais forte entre as HS e o suporte de pares, percebe-se que o suporte familiar continua sendo uma fonte importante de apoio (Nilsen et al., 2013; Pinto et al., 2016; Schoenrock et al., 1999). O suporte parental esteve positivamente relacionado às HS em crianças, corroborando Braz et al. (2013) e Cômodo et al. (2017), que afirmaram que as relações de intimidade entre pais e filhos, construídas a partir da expressão de sentimentos, da comunicação e do estabelecimento de limites e regras (Murta et al., 2013), são relevantes para o desenvolvimento saudável das relações interpessoais dos filhos.

Considerando que, ao longo do tempo, o suporte social recebido como consequência do uso de HS também pode aumentar a emissão das HS em momentos posteriores, vale notar que adolescentes que relataram possuir mais suporte social de amigos avaliaram mais positivamente o seu próprio desempenho social (Bédard et al., 2014; Eskin, 2003; García-Bóveda et al., 2007; Kukulu et al., 2006). Esse dado pode indicar que, por muitas das descobertas entre jovens estarem ligadas 
ao contexto escolar e social, como relacionamentos amorosos, participação em festas, escolha profissional, e outros (Nilsen et al., 2013), o contexto social envolvendo pares tem influência sobre a aquisição e a funcionalidade das HS. Assim, adolescentes que não recebem o suporte de pares podem passar por uma fase com maior probabilidade de isolamento e depressão (Del Prette \& Del Prette, 2017; Nilsen et al., 2013).

No que diz respeito a diferenças ligadas ao sexo, em quatro estudos (Bédard et al., 2014; Eskin, 2003; Nilsen et al., 2013; Schoenrock et al., 1999), a relação entre as HS e o suporte social foi examinada separadamente para pessoas do sexo feminino e masculino. Em todos esses estudos, as correlações entre as HS e o suporte social foram mais fortes entre pessoas do sexo masculino do que do sexo feminino, e todos os participantes desses estudos eram adolescentes. Isso pode significar: (a) que havia menor variação entre as meninas do que entre os meninos em relação aos seus escores em HS ou na obtenção de suporte social, ou ambos; ou (b) que o uso de HS foi menos útil para obter suporte social para as meninas do que para os meninos. Assim, existem estudos sobre as HS que discutem gênero (Mackin et al., 2017; Malinauskas et al., 2014; Martins et al., 2014; Santos, 2016), mas ainda é necessário investigar por que ocorre a diferença entre os resultados observados entre meninos e meninas.

Nota-se também, na Tabela 2, que o medo de críticas, de contato social e a incapacidade de negar pedidos estiveram negativamente relacionados com suporte social (Dierk et al., 2006). Isto é, indivíduos que apresentaram déficits nessas classes de HS perceberam menor nível de suporte social. Pessoas que não conseguem ser assertivas, negando pedidos abusivos, por exemplo, ou ainda se esquivando de contato social ou tendo medo de receber críticas (mesmo justas), podem ter mais dificuldades de desenvolver relacionamentos de boa qualidade, o que implicaria em maior dificuldade de receber suporte social, quando necessário. Segundo Del Prette e Del Prette (2017), é importante que o indiví- duo saiba receber críticas acerca de seu desempenho, sendo esta uma oportunidade para autorregular os seus comportamentos e estabelecer relações interpessoais de maior qualidade. Além disso, é necessário que seja habilidoso em negar pedidos, pois aceitar pedidos sem genuinamente querer fazê-lo pode indicar um desempenho social pouco competente por existir uma incompatibilidade entre o fazer e o sentir (Del Prette \& Del Prette, 2017). De maneira geral, esses déficits em receber críticas e negar pedidos tendem a aumentar a probabilidade de isolamento e medo de contato social (Del Prette \& Del Prette, 2017).

Além disso, tendo em vista que alguns dos pesquisadores analisaram a influência de HS mais específicas sobre a obtenção de suporte social, é possível notar que as HS envolvendo assertividade (por parte de adolescentes) parecem influenciar a obtenção de suporte de pares (Kululu et al., 2006). Em outros estudos, foi possível confirmar que déficits em algumas HS (medo de críticas, incapacidade de negar pedidos, medo de contato social) estiveram significativamente relacionados com menor apoio recebido e menor integração social (Bédard et al., 2014; Carneiro et al., 2007; Nilsen et al., 2013; Segal, 2005; Segrin et al., 2016). Déficits em HS e em competência social podem influenciar a capacidade de acessar e manter a rede de apoio, aumentando a vulnerabilidade das pessoas ao sofrimento psicológico porque têm menos acesso aos efeitos protetores do suporte social, como o apoio emocional (Neri, 2014; Segrin et al., 2016).

Ao se considerar a relação entre HS e o tamanho da rede de apoio, García-Bóveda et al. (2007) e Riggio e Kwong (2009) encontraram uma correlação fraca, porém estatisticamente significativa, entre essas variáveis. Assim, um repertório mais amplo de HS pode indicar uma rede de apoio com mais membros. Porém, dada a baixa magnitude da correlação entre essas variáveis, infere-se que outros aspectos possam interferir nessa relação, como a origem do suporte, a função desse e a satisfação com o apoio recebido (Bédard et al., 2014; García-Bóveda et al., 2007; Riggio \& Kwong, 2009) 


\section{Considerações finais}

Em conclusão, o objetivo do presente estudo foi investigar fatores que afetam a magnitude da relação entre as HS e o suporte social, uma vez que tanto as HS quanto o suporte social têm efeito benéfico para as relações interpessoais e a saúde mental dos indivíduos, como por exemplo, menor presença de depressão, ansiedade, estresse, sentimento de solidão e, consequentemente, uma melhor qualidade de vida. Com base nesta revisão sistemática de literatura realizada, observou-se que as HS e o suporte social apresentaram correlação positiva e de magnitude fraca a moderada e que os principais fatores que parecem influenciar a magnitude da relação entre essas variáveis são: (a) faixa etária e fonte de apoio, uma vez que, para adolescentes e crianças, há uma influência maior das HS sobre o apoio de pares, mas menor sobre o apoio de familiares; e (b) sexo, pois observou-se uma associação mais forte entre as HS e o apoio social para adolescentes do sexo masculino do que do sexo feminino. Além disso, destaca-se a relevância das habilidades de assertividade na obtenção de suporte social.

Dado o exposto, afirma-se que a principal contribuição da presente revisão foi oferecer um suporte teórico que reforça a importância da elaboração de treinamentos de HS para indivíduos que apresentam déficits nessas habilidades, pois em pesquisas de intervenção com diferentes populações, estudiosos demonstraram que a aprendizagem de HS pode contribuir para o aumento do suporte social percebido e recebido (Leme, Fernandes, Jovarini, Achkar, \& Del Prette, 2016; Segrin et al., 2016). Assim, treinamentos em HS específicas para cada população de interesse podem gerar efeitos positivos nos níveis de suporte social percebido e, consequentemente, na qualidade de vida de diferentes populações, confirmando mais uma vez as hipóteses iniciais deste estudo.
Entendendo que a presente revisão é um recorte da literatura sobre as HS e o suporte social, mencionam-se como limitações deste estudo o fato de a pesquisa ter sido realizada em apenas três idiomas e apenas com artigos de acesso livre ou que as autoras tiveram acesso via as bases de dados disponibilizadas pela universidade, $\mathrm{o}$ que pode ter limitado a obtenção de outros estudos com informações importantes. Neste estudo, por exemplo, não foi possível descobrir a influência de outras variáveis sociodemográficas (tais como a escolaridade), ou informações mais detalhadas sobre as classes de HS, além da assertividade, que estão diretamente relacionadas ao acesso e, especialmente, à manutenção do suporte social. Mesmo assim, com base no conjunto de resultados examinado, foi possível identificar algumas variáveis intervenientes que podem influenciar na probabilidade que o uso de habilidades sociais resultará na obtenção de apoio social. Para confirmar essas observações, será importante testar um modelo teórico sobre a relação entre as HS e o suporte social, relacionando todas as variáveis encontradas na presente revisão, considerando variáveis de contexto social (apoio de pares ou de familiares, em situações sociais normativas e não normativas), mediadoras (idade, sexo) e de desfecho (indicadores de saúde mental e bem-estar).

\section{Referências}

Anfara, V. A., Brown, K. M., \& Mangione, T. L. (2002). Qualitative analysis on stage: Making the research process more public. Educational Researcher, 31, 28-38. doi: 10.3102/0013189x031007028

*1Bédard, K., Bouffard, T., \& Pansu, P. (2014). The risks for adolescents of negatively biased self-evaluations of social competence: The mediating role of social support. Journal of Adolescence, 37(6), 787-798. doi: 10.1016/j. adolescence.2014.05.004

Bolsoni-Silva, A. T., \& Loureiro, S. R. (2014). The role of social skills in social anxiety of university

As referências bibliográficas sinalizadas com um asterisco (*) fazem parte do corpo de estudos examinado para a presente revisão de literatura. 
students. Paidéia, 24(58), 223-232. doi: 10.1590/198243272458201410

Braz, A. C., Cômodo, C. N., Del Prette, Z. A., Del Prette, A., \& Fontaine, A. M. G. (2013). Habilidades sociales e intergeneracionalidad en las relaciones familiares. Apuntes Psicología, 31(1), 77-84. Retrieved from http://apuntesdepsicologia.es/index.php/revista/ article/view/305 https://doi.org/10.5380/psi.v21i2.50314 Campos, J. R., Del Prette, Z. A. P., \& Del Prette, A. (2018). Relações entre depressão, habilidades sociais, sexo e nível socioeconômico em grandes amostras de adolescentes. Psicologia: Teoria e Pesquisa, 34, 1-10. doi: 10.1590/0102.3772e3446

Cardoso, H. F., \& Baptista, M. N. (2015). Evidência de validade para a Escala de Percepção do Suporte Social (Versão Adulta) - EPSUS-A: Um estudo correlacional. Psicologia: Ciência e Profissão, 35(3), 946-958. doi: 10.1590/1982-3703001352013

*Carneiro, R. S., Falcone, E., Clark, C., Del Prette, Z., \& Del Prette, A. (2007). Qualidade de vida, apoio social e depressão em idosos: Relação com habilidades sociais. Psicologia: Reflexão e Crítica, 20(2), 229-237. doi: 10.1590/s0102-79722007000200008

Carneiro, R. S. (2014). Um estudo das habilidades sociais em idosos. Psicologia Argumento, 32(76), 23-31. https:// doi.org/10.7213/psicolargum.v32i76.19747

Carneiro, R. S., \& Falcone, E. F. (2016). Avaliação de um programa de promoção de habilidades sociais para idosos. Análise Psicológica, 34(3), 279-291. doi: 10.14417/ap.960

Cômodo, C. N., Del Prette, A., \& Del Prette, Z. A. P. (2017). Intergeracionalidade das habilidades sociais entre pais e filhos adolescentes. Psicologia: Teoria e Pesquisa, 33, 1-9. doi: 10.1590/0102.3772e33311

Del Prette, A., \& Del Prette, Z. A. P (2009). Inventário de Habilidades Sociais para Adolescentes (IHSA-Del Prette): Manual de Aplicação, Apuração e Interpretação. São Paulo: Casa do Psicólogo. https://doi.org/10.12957/ epp.2011.8389

Del Prette, Z. A. P., \& Del Prette, A. (2017). Habilidades Sociais e Competência Social para uma Vida Melhor. São Carlos, SP: EDUFSCar. https://doi. org/10.7476/9786580216161
*Dierk, J. M., Conradt, M., Rauh, E., Schlumberger, P., Hebebrand, J., \& Rief, W. (2006). What determines well-being in obesity? Associations with BMI, social skills, and social support. Journal of Psychosomatic Research, 60(3), 219-227. doi: 10.1016/j.jpsychores.2005.06.083

*Eskin, M. (2003). Self-reported assertiveness in swedish and turkish adolescents: A cross-cultural comparison. Scandinavian Journal of Psychology, 44(1), 7-12. doi:10.1111/1467-9450.t01-1-00315

*García-Bóveda, R. J., Morejón, A. J. V., \& Jiménez, R. V. M. (2007). Habilidades sociales y apoyo social en esquizofrenia. Revista Internacional De Psicología y Terapia Psicológica, 7(1), 61-71. Retrieved from http:// www.redalyc.org/html/560/56070105/

Giron, L. U., \& de Souza, M. S. V. (2015). Habilidades sociais: Fator de proteção contra transtornos alimentares em adolescentes. Revista Ciência \& Saúde Coletiva, 20(11), 3495- 3508. doi: 10.1590/1413812320152011.18362014

Hajek, A., Brettschneider, C., Lange, C., Posselt, T., Wiese, B., Steinmann, S.,... König, H.H. (2016). Gender differences in the effect of social support on health-related quality of life: results of a population-based prospective cohort study in old age in Germany. Quality of Life Research, 25(5), 1159-1168. doi: 10.1007/s11136015-1166-5

Hill, E. M. (2016). Quality of life and mental health among women with ovarian cancer: Examining the role of emotional and instrumental social support seeking. Psychology, Health \& Medicine, 21(5), 551-561. doi: 10.1080/13548506.2015.1109674

*Kukulu, K., Buldukoğlu, K., Kulakaç, Ö., \& Köksal, C. D. (2006). The effects of locus of control, communication skills and social support on assertiveness in female nursing students. Social Behavior and Personality: An International Journal, 34(1), 27-40. doi: 10.2224/ sbp.2006.34.1.27

Leme, V. B. R., Fernandes, L. D. M., Jovarini, N. V., Achkar, A. M. E., \& Del Prette, Z. A. P. (2016). Social skills program for adolescents in vulnerable social contexts. Psico-USF, 21(3), 595-608. doi: 10.1590/141382712016210313

Levin, J., \& Fox, J. A. (2004). Estatística para Ciências Humanas. São Paulo, SP: Pearson. 
Mackin, D. M., Perlman, G., Davila, J., Kotov, R., \& Klein, D. N. (2017). Social support buffers the effect of interpersonal life stress on suicidal ideation and self-injury during adolescence. Psychological Medicine, 47(6), 1149-1161. doi: 10.1017/S0033291716003275

Malinauskas, R., Dumciene, A., \& Lapeniene, D. (2014). Social skills and life satisfaction of Lithuanian first-and senior-year university students. Social Behavior and Personality: An International Journal, 42(2), 285-293. doi: 10.2224/sbp.2014.42.2.285

Martins, R. P., Nunes, S. A. N., Faraco, A. M. X., Manfroi, E. C., Vieira, M. L., \& Rubin, K. H. (2014). Práticas parentais: Associações com desempenho escolar e habilidades sociais. Psicologia Argumento, 32(78), 89-100. https://doi.org/10.7213/psicol.argum.32.078.ao04

*McIntosh, R. C., Ironson, G., Antoni, M., Fletcher, M. A., \& Schneiderman, N. (2016). Alexithymia, assertiveness and psychosocial functioning in HIV: Implications for medication adherence and disease severity. AIDS and Behavior, 20(2), 325-338. doi: 10.1007/s10461-015-1126-7

*Müller, R., Peter, C., Cieza, A., Post, M. W., Van Leeuwen, C. M., Werner, C. S., \& Geyh, S. (2015). Social skills: A resource for more social support, lower depression levels, higher quality of life, and participation in individuals with spinal cord injury? Archives of Physical Medicine and Rehabilitation, 96(3), 447-455. doi: 10.1016/j.apmr.2014.09.006

Murta, S. G., Del Prette, A., \& Del Prette, Z. A. (2013). Prevenção ao sexismo e ao heterosexismo entre adolescentes: Contribuições do treinamento em habilidades de vida e habilidades sociais. Revista de Psicologia da Criança e do Adolescente, 1(2), 73-85. Retrieved from http://revistas.lis.ulusiada.pt/index.php/rpca/article/ view/21 https://doi.org/10.5380/psi.v21i2.50314

Neri, A. L. (2014). Palavras-chave em Gerontologia (4a ed.). Campinas, SP: Editora Alínea.

*Nilsen, W., Karevold, E., Røysamb, E., Gustavson, K., \& Mathiesen, K. S. (2013). Social skills and depressive symptoms across adolescence: Social support as a mediator in girls versus boys. Journal of Adolescence, 36(1), 11-20. https://doi.org/10.1016/j.adolescence.2012.08.005

Pasquali, L. (2015). Delineamento de Pesquisa em Ciência. Vol. 2. São Paulo, SP: Vetor.
Pinquart, M., \& Sörensen, S. (2000). Influences of socioeconomic status, social network, and competence on subjective well-being in later life: A meta-analysis. Psychology and Aging, 15(2), 187-224. doi: 10.1037/08827974.15.2.187

Pinto, F. N. F. R., \& Oliveira, D. C. (2015). Capacidade funcional e envolvimento social em idosos: Há relação? Revista Brasileira de Ciências do Envelhecimento Humano, 12(1), 56-68. doi: 10.5335/rbceh.v12i1.4687

Pinto, F. N. F. R. (2016). Construção e análise psicométrica de um inventário de habilidades sociais para cuidadores de idosos familiares. Tese de Doutorado. Universidade Federal de São Carlos, São Carlos-SP, 106 pp. Retrieved from https://repositorio.ufscar.br/ handle/ ufscar/7997?show=full https://doi.org/10.5327/ z2447-211520171700046

Pinto, F. N. F. R., Barham, E. J., \& Del Prette, Z. A. P. (2016). Interpersonal conflicts among family caregivers of the elderly: The importance of social skills. Paidéia, 26(64), 161-170. doi: 10.1590/1982-43272664201605

Queluz, F. N. F. R., Barham, E. J., Del Prette, Z. A. P., \& Santos, A. A. A. (2018). Social Skills Inventory for Caregivers of Elderly Family Members (SSI-CE): Relations with indicators of psychological wellbeing. Trends in Psychology, 26(2), 551-563. doi: 10.9788/ TP2018.2-01En

*Riggio, H. R., \& Kwong, W. Y. (2009). Social skills, paranoid thinking, and social outcomes among young adults. Personality and Individual Differences, 47(5), 492-497. doi: 10.1016/j.paid.2009.04.026

Rodrigues, V. B., \& Madeira, M. (2009). Suporte Social e saúde mental: Revisão da literatura. Revista da Faculdade de Ciências da Saúde, 6, 390-399. Retrieved from http://bdigital.ufp.pt/handle/10284/1293 https:// doi.org/10.4034/rbcs.2011.15.04.16

Santos, L. A. D. (2016). Diferenças de Gênero no Suporte Social, Ajustamento Emocional e Satisfação com a Vida em Estudantes Universitários do $1^{\circ}$ ano (Dissertação de mestrado). Universidade Portucalense, Portugal. Retrieved from http://hdl.handle.net/11328/1630

*Segal, D. L. (2005). Relationships of assertiveness, depression, and social support among older nursing home residents. Behavior Modification, 29(4), 689-695. doi: $10.1177 / 0145445503259391$ 
*Segrin, C., McNelis, M., \& Swiatkowski, P. (2016). Social skills, social support, and psychological distress: A test of the social skills deficit vulnerability model. Human Communication Research, 42(1), 122-137. doi: 10.1111/hcre. 12070

*Schoenrock, C. J., Bell, N. J., Sun, S. W., \& Avery, A. W. (1999). Family correlates of adolescent self-monitoring and social competence. The Journal of Psychology, 133(4), 377-393. doi: 10.1080/00223989909599750

Stasiak, G. R., Weber, L. N. D., \& Tucunduva, C. (2014). Qualidade na interação familiar e estresse parental e suas relações com o autoconceito, habilidades sociais e problemas de comportamento dos filhos. Psico, 45(4), 494-501. doi: 10.15448/1980-8623.2014.4.15846

*Takahashi, Y., Okada, K., Hoshino, T., \& Anme, T. (2015). Developmental trajectories of social skills during early childhood and links to parenting practices in a Japanese sample. PloS One, 10(8), doi: 10.1371/journal. pone. 0135357

*Vázquez, M. G., \& Lemos, V. N. (2013). Habilidades sociales y apoyo social percibido en niños con diagnóstico. Acta Psiquiátrica y Psicológica de América Latina, 59(2), 87-93. Retrieved from: http://ri.conicet. gov.ar/handle/11336/26583

\section{Endereço para correspondência: Vanessa Santiago}

Ximenes (Avenida São Paulo, 550, apto 1402, Londrina, Paraná, 86010-060; (43) 99635-7881; vanessasximenes@hotmail.com).

Recebido em: 8/7/2018.

Aprovado em: 14/5/2019.

Publicado em: xx/xx/2019.

\section{Vanessa Santiago Ximenes}

E-mail: vanessasximenes@hotmail.com

Titulação Acadêmica: Mestre em Psicologia

Afiliação Institucional: Universidade Federal de São

Carlos

\section{Francine Náthalie Ferraresi Rodrigues Queluz}

E-mail: francine.queluz@gmail.com

Titulação Acadêmica: Pós-doutoranda em Psicologia Afiliação Institucional: Universidade São Francisco

\section{Elizabeth Joan Barham}

E-mail: lisa.barham.ufscar@gmail.com

Titulação Acadêmica: Pós-doutora em Psicologia

Afiliação Institucional: Universidade Federal de São

Carlos 